\title{
A low-complexity AFF-RLS algorithm using a normalization technique
}

\author{
Seokjin Lee ${ }^{1 \mathrm{a})}$, Jun-seok Lim² ${ }^{2}$, and Koeng-Mo Sung ${ }^{1}$ \\ ${ }^{1}$ Applied Acoustics Lab., Institute of New Media and Communications, \\ Department of Electrical Engineering, Seoul National University, \\ San 56-1, Sillim-dong, Kwanak-gu, Seoul 151-742, Republic of Korea \\ ${ }^{2}$ Department of Electronics Engineering, Sejong University, \\ 98 Gunja-Dong, Gwangjin-Gu, Seoul 143-747, Republic of Korea \\ a) sjlee@acoustics.snu.ac.kr
}

\begin{abstract}
In this paper, we propose a modified RLS algorithm with Adaptive Forgetting Factor using a low-complexity forgetting factor update equation. The conventional AFF-RLS method has a highcomplexity update equation to update forgetting factor. In order to reduce complexity, an approximated version of the AFF-RLS method had been derived by Song. But this modified AFF-RLS method shows degraded performance because it suffers from 'gradient error amplification' problem. In order to obtain the same performance as AFF-RLS with relatively low computational cost, we noted that AFF-RLS had been derived by 'method of steepest descent', and we use normalization technique which is used in NLMS. The Experiment result shows that the proposed method has almost same performance as the conventional AFF-RLS method with relatively low-complexity.
\end{abstract}

Keywords: RLS, AFFRLS, VFFRLS, adaptive filter

Classification: Science and engineering for electronics

\section{References}

[1] S. Haykin, Adaptive Filter Theory, Fourth edition, Prentice Hall, New Jersey, pp. 662-663, 2002.

[2] S. Song, "Self-Tunning Adaptive Algorithm and Applications," Ph. D. Dissertation, Seoul National University, pp. 15-25, Aug. 2003. [Online] http://library.snu.ac.kr/Eng/DetailView.jsp?uid=11\&cid=1112838

[3] S. Haykin, Adaptive Filter Theory, Fourth edition, Prentice Hall, New Jersey, pp. 436-443, 2002.

[4] S. Haykin, Adaptive Filter Theory, Fourth edition, Prentice Hall, New Jersey, pp. 203-204, 2002.

[5] S. Haykin, Adaptive Filter Theory, Fourth edition, Prentice Hall, New Jersey, pp. 320-324, 2002.

[6] S. Haykin, Adaptive Filter Theory, Fourth edition, Prentice Hall, New Jersey, pp. 120-125, 2002. 


\section{Introduction}

The RLS (Recursive Least Squares) method is a broadly used adaptive algorithm for signal processing in electronic engineering. The RLS algorithm shows a good performance and a fast adaptation within a stationary environment, but it shows a poor performance within a non-stationary environment because of the fixed forgetting factor.

In order to enhance the 'tracking' performance, RLS methods with an adaptive forgetting factor need to be developed. One typical method is described in Haykin's book [1]. This method shows a good tracking performance, however, it suffers from heavy computational loads. In order to overcome the disadvantages, Song [2] proposed the MAFF-RLS (Modified AFF-RLS, AFF-RLS means 'Adaptive Forgetting Factor - RLS') method. However, this method shows a poor performance because of forgetting factor approximation error.

In this paper, we propose an improved MAFF-RLS method in order to overcome the above problem. In order to achieve this purpose, we will draw an analogy between the LMS (Least Mean Squares) and the AFF-RLS method, and use the normalization technique found in the LMS method.

\section{The Conventional AFF-RLS method}

\subsection{The RLS method}

The RLS (Recursive Least Squares) method is the recursive algorithm of the least squares method. The detail of the RLS method is explained in [3]. In summary, the RLS method can be represented by [3]:

$$
\begin{gathered}
\underline{k}(n)=\frac{P(n-1) \underline{u}(n)}{\lambda+\underline{u}^{H}(n) P(n-1) \underline{u}(n)}, \\
\xi(n)=d(n)-\underline{\hat{w}}^{H}(n-1) \underline{u}(n), \\
\underline{\hat{w}}(n)=\underline{\hat{w}}(n-1)+\underline{k}(n) \xi^{*}(n), \\
P(n)=\lambda^{-1} P(n-1)-\lambda^{-1} \underline{k}(n) \underline{u}^{H}(n) P(n-1),
\end{gathered}
$$

where $\underline{k}(n)$ is a gain vector, $\xi(n)$ is a priori estimation error, $\underline{\hat{w}}(n)$ is the weight vector, and $P(n)$ is the inversion of the correlation matrix.

\subsection{The AFF-RLS method}

The forgetting factor $\lambda$ is a constant in the conventional RLS method. In the Adaptive Forgetting Factor RLS (AFF-RLS) method, $\lambda$ is updated recursively in order to adjust itself to non-stationary circumstances.

The goal of the AFF-RLS method is to calculate $\lambda$ which optimizes the mean squares of a priori estimation error. Therefore, the cost function is determined as [1]:

$$
J^{\prime}(n)=\frac{1}{2} E\left[|\xi(n)|^{2}\right]
$$

where $\xi(n)$ is a priori estimation error referred to above. 
For the optimization, take the partial derivative of the cost function with respect to $\lambda$.

$$
\begin{aligned}
\nabla_{\lambda}(n) & =\frac{\partial J^{\prime}(n)}{\partial \lambda}=\frac{1}{2} E\left[\frac{\partial \xi(n)}{\partial \lambda} \xi^{*}(n)+\frac{\partial \xi^{*}(n)}{\partial \lambda} \xi(n)\right] \\
& =-\frac{1}{2} E\left[\psi^{H}(n-1) u(n) \xi^{*}(n)+u^{H}(n) \psi(n-1) \xi(n)\right]
\end{aligned}
$$

where $\psi(n)=\frac{\partial \hat{w}(n)}{\partial \lambda}$ and $S(n)=\frac{\partial P(n)}{\partial \lambda}$.

According to the "method of steepest descent," we may adaptively compute the forgetting factor using the recursion [1]:

$$
\begin{aligned}
\lambda(n) & =\lambda(n-1)-\alpha \hat{\nabla}_{\lambda}(n) \\
& =\lambda(n-1)+\alpha \operatorname{Re}\left[\underline{\psi}^{H}(n-1) \underline{u}(n) \xi^{*}(n)\right],
\end{aligned}
$$

where $\alpha$ is a small, positive learning-rate parameter.

In summary, the forgetting factor update process of the AFF-RLS method can be stated as [1]:

$$
\begin{gathered}
S(n)=[\lambda(n-1)]^{-1}\left[I-\underline{k}(n) \underline{u}^{H}(n)\right] S(n-1)\left[I-u(n) k^{H}(n)\right] \\
+[\lambda(n-1)]^{-1} \underline{k}(n) \underline{k}^{H}(n)-[\lambda(n-1)]^{-1} P(n), \\
\underline{\psi}(n)=\left[I-\underline{k}(n) \underline{u}^{H}(n)\right] \underline{\psi}(n-1)+S(n) \underline{u}(n) \xi^{*}(n), \\
\lambda(n)=\lambda(n-1)+\left.\alpha \operatorname{Re}\left[\underline{\psi}^{H}(n-1) \underline{u}(n) \xi^{*}(n)\right]\right|_{\lambda_{-}} ^{\lambda_{+}} .
\end{gathered}
$$

\section{The NAFF-RLS and its Approximation}

\subsection{The normalization technique in the NLMS}

The LMS algorithm can start from the "method of steepest descent." According to this method, the weight vector can be updated as [4]:

$$
\hat{w}(n+1)=\hat{w}(n)-\frac{\mu}{2} \nabla J(n),
$$

where $\underline{\hat{w}}(n)$ is a weight vector at the time $n, \nabla J(n)$ is a gradient of cost function $J(n)$ and $\mu$ is a step-size parameter. According to [4], the gradient of the cost function in the LMS is

$$
\nabla J(n)=-2 \underline{p}+2 R \underline{w}(n) \approx-2 \underline{u}(n) e^{*}(n),
$$

where $R=E\left[\underline{u u}^{H}\right]$ and $p=E\left[\underline{u} d^{*}\right]$. Using the 'method of steepest descent',

$$
\hat{w}(n+1)=\hat{w}(n)-\frac{\mu}{2} \nabla J(n)=\hat{w}(n)+\mu \underline{u}(n) e^{*}(n) .
$$

As shown in Eq. (13), the adjustment is directly proportional to the tapinput vector $\underline{u}(n)$. Therefore, when $\underline{u}(n)$ is large, the LMS filter suffers from a 'gradient noise amplification' problem. In order to overcome this difficulty, we can use the normalized LMS (NLMS) filter [5].

In order to avoid the 'gradient noise amplification' problem, the added term to the priori weight vector is normalized with the norm of the input vector. According to [5], the weight vector update equation of the NLMS is:

$$
\underline{\hat{w}}(n+1)=\underline{\hat{w}}(n)+\frac{\tilde{\mu}}{\|\underline{u}(n)\|^{2}} \underline{u}(n) e^{*}(n) .
$$


Table I. The analogy between the forgetting factor update equation of the RLS and the filter weight update equation of the LMS.

\begin{tabular}{|l||l|l|}
\hline & $\begin{array}{l}\text { LMS Weight Vector } \\
\text { Update Equation }\end{array}$ & $\begin{array}{l}\text { AFF-RLS Forgetting Factor } \\
\text { Update Equation }\end{array}$ \\
\hline \hline$z(n)$, updated term & $\underline{\hat{w}}(n)$ & $\lambda(n)$ \\
\hline$c_{\text {step }}$, step-size & $\mu$ & $\alpha$ \\
\hline$\varepsilon$, error in cost function & $e(n)$ & $\xi(n)$ \\
\hline$s_{\text {scaling }}$, gradient amp. term & $\underline{u}(n)$ & $\psi^{H}(n-1) \underline{u}(n)$ \\
\hline
\end{tabular}

\subsection{The NAFF-RLS}

The AFF-RLS method and the NLMS method are stated above. In order to derive the NAFF-RLS method, we have to note that ' $\underline{\hat{w}}(n)$ recursion of the LMS' and ' $\lambda$ recursion of the AFF-RLS' are derived using the steepest descent algorithm. Therefore, it can be inferred that an analogy between the recursion of the LMS and the AFF-RLS exists.

As previously shown, both recursion equations consist of the same structure as shown in Table I. This analogy may not have any physical meaning, but it can be useful in the development of a method which overcomes 'the gradient noise amplification' problem of the forgetting factor recursion.

Using this analogy, the same normalizing technique used in the NLMS method can be used in the forgetting factor update equation of the Normalized AFF-RLS (NAFF-RLS) method and can be derived as:

$$
\lambda(n)=\lambda(n-1)+\left.\frac{\alpha}{\left|\psi^{H}(n-1) \underline{u}(n)\right|^{2}} \operatorname{Re}\left[\psi^{H}(n-1) \underline{u}(n) \xi^{*}(n)\right]\right|_{\lambda_{-}} ^{\lambda_{+}},
$$

where the meaning of each of the terms is the same as Eq. (7), and $\lambda+, \lambda_{-}$ are the upper and lower limit of the forgetting factor.

The NAFF-RLS is more effective when it is modified to the approximated version because the normalization can prevent the amplification of the approximation error. The approximated versions of the NAFF-RLS method will be stated in the next section.

\subsection{The approximation of the NAFF-RLS}

The AFF-RLS method shows a good performance, but it suffers from heavy computational loads. In order to reduce the computational cost, an approximated version of the AFF-RLS can be derived with following approximations [2].

According to [2],

$$
C(n)=I-\underline{k}(n) \underline{u}^{H}(n) \approx c(n) I,
$$

where $c(n)=1-\frac{\underline{u}^{H}(n) \underline{\underline{k}}(n)}{L}$. This approximation can be possible because $E[C(n)]$ is a diagonal matrix. The detailed proof of the above approximation is shown in [2]. Because there are so many $C(n)$ calculations in the 
AFF-RLS method, this approximation can reduce the number of complex multiplications. Using this approximation, the $\hat{S}(n), \underline{\hat{\psi}}(n)$, and $\hat{\lambda}(n)$ can be calculated with low-complexity. This method is called as MAFF-RLS (Modified AFF-RLS).

However, this approximation makes an estimation error in $\nabla_{\lambda}(n)$. Therefore, this MAFF-RLS method will severely suffer from the 'gradient error amplification' problem. In order to resolve this problem, we can use the MNAFF-RLS method rather than the MAFF-RLS method.

The MNAFF-RLS (Modified NAFF-RLS) method can be derived by applying the same approximation (Eq. (16)) to the NAFF-RLS. In summary, the proposed MNAFF-RLS method consists of:

$$
\begin{gathered}
\underline{k}(n)=\frac{P(n-1) \underline{u}(n)}{\hat{\lambda}_{n}(n-1)+\underline{u}^{H}(n) P(n-1) \underline{u}(n)}, \\
\xi(n)=d(n)-\hat{w}^{H}(n-1) u(n), \\
\hat{w}(n)=\hat{w}(n-1)+k(n) \xi^{*}(n), \\
P(n)=\frac{P(n-1)-k(n) \underline{u}^{H}(n) P(n-1)}{\hat{\lambda}_{n}(n-1)}, \\
\hat{S}_{n}(n)=\left[\hat{\lambda}_{n}(n-1)\right]^{-1}|c(n)|^{2} \hat{S}(n-1)-\left[\hat{\lambda}_{n}(n-1)\right] \\
\underline{\hat{\psi}}_{n}(n)=c(n) \underline{\hat{\psi}}_{n}(n-1)+\hat{S}_{n}(n) \underline{u}(n) \xi^{*}(n), \\
\hat{\lambda}_{n}(n)=\hat{\lambda}_{n}(n-1)+\frac{\alpha}{\left.\left|\hat{\psi}_{n}^{H}(n-1) \underline{u}(n)\right|^{2} \operatorname{Re}\left[\underline{\hat{\psi}}_{n}^{H}(n-1) \underline{u}(n) \xi^{*}(n)\right]\right|_{\lambda_{-}} ^{\lambda_{+}} .}
\end{gathered}
$$

The number of complex multiplications of each method is shown in Table II where $L$ is the filter length.

\section{Simulation}

\subsection{The Adaptive Beamformer for Moving Interference Can- cellation}

In order to evaluate the performance of the methods, each method was applied to an adaptive beamformer. As shown in Fig. 1 (a), each method was used for adjusting the weight vector of the beamformer. In our simulations we used the GSC (Generalized Sidelobe Canceller) for moving interference cancellation. The details of the GSC are described in [6]. In our simulation scenario one beamformer, one desired signal, and one moving interference signal were present, as shown in Fig. 1 (b).

\subsection{The Settings}

The beamformer had 12 sensors, arrayed in a line. And the input signal sampling frequency was $1000 \mathrm{~Hz}$. The desired sensor signal incident was on the front $\left(\theta_{s}=0^{\circ}\right)$, and the moving interference source started from the left at an angle of $45^{\circ}$ and moved to the right at an angle of $45^{\circ}$ for 1000 milliseconds, 
Table II. The Comparison of the Computational Costs.

\begin{tabular}{|l|l|l|}
\hline & $\begin{array}{l}\text { Complex } \\
\text { Multiplication }\end{array}$ & $\begin{array}{l}\text { Additional complexity apart } \\
\text { from conventional RLS }\end{array}$ \\
\hline RLS & $2.5 L^{2}+3 L$ & 0 \\
\hline AFF-RLS & $9 L^{2}+7 L$ & $6.5 L^{2}+4 L$ \\
\hline MAFF-RLS & $4 L^{2}+5 L$ & $1.5 L^{2}+2 L$ \\
\hline MNAFF-RLS & $4 L^{2}+6 L$ & $1.5 L^{2}+3 L$ \\
\hline
\end{tabular}

following a line parallel to the sensor array, as shown in Fig. 1 (b). And both signals - the desired source and the interference signal - were in the far-field.

In our simulations, $\lambda$ in the conventional RLS was 0.99 , and the 'learningrate' $\alpha$ in the AFF-RLS, the MAFF-RLS, and the MNAFF-RLS was 0.02.

A SINR (Signal-to-Noise-and-Interference-Ratio) was used to measure the performance in this experiment, which denotes the ratio between the desired signal energy to the noise and the interference signal energy. The formula expression of the SINR is:

$$
\operatorname{SINR}(t)=\frac{\left|\underline{w}^{H}(t) \underline{y}_{s}(t)\right|^{2}}{E\left\{\left|\underline{w}^{H}(t) \underline{y_{i}}(t)\right|^{2}\right\}}=\frac{P_{s}\left|\underline{w}^{H}(t) \underline{a}\left(\theta_{s}\right)\right|^{2}}{\underline{w}^{H}(t) R_{i+n}(t) \underline{w}(t)} .
$$

\subsection{The Result}

The simulation results are shown in Fig. $1(\mathrm{c})$ and (d). The x-axis of the graph represents a sampling time. The y-axis of the Fig. 1 (c) graph denotes the SINR in dB. In the graph, the better method shows higher SINR values and the instantaneous performance is less important. And the result of the NAFF-RLS is excluded because it is almost the same as the result of the AFF-RLS.

In Fig. 1 (c), the SINR curves of all the methods have a minimum value when the snapshot index is 500 because the DOA (Direction Of Arrival) of the interference and of the desired sources are the same. As shown in Fig. 1 (c), the performance of the MNAFF-RLS is almost same as that of the AFF-RLS. The purpose of the MNAFF-RLS is to approximate the AFFRLS with a relatively low computation cost, so the result of the MNAFFRLS in the Fig. 1(c) satisfies the purpose for which the MNAFF-RLS was developed. Furthermore, the performance of the MNAFF-RLS is shown to be much better than the MAFF-RLS after the valley.

The changes to the forgetting factor of each method are shown in Fig. 1 (d). As shown in Fig. 1(d), the forgetting factor of the MNAFF-RLS approximates that of the AFF-RLS more precisely than that of the MAFF-RLS.

\section{Conclusion}

In this paper, the MNAFF-RLS are developed and evaluated. The NAFFRLS is developed using the analogy between the NLMS and the AFF-RLS, and the MNAFF-RLS is a derived form of the NAFF-RLS method. 
The MNAFF-RLS method was adopted in an adaptive beamformer for moving interference cancellation, and evaluated. The simulation result showed that the proposed MNAFF-RLS is better than the MAFF-RLS method and the MNAFF-RLS has a very similar performance to the AFF-RLS method.

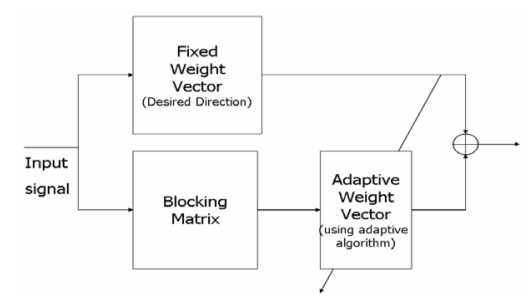

(a) The adaptive GSC to evaluate each method

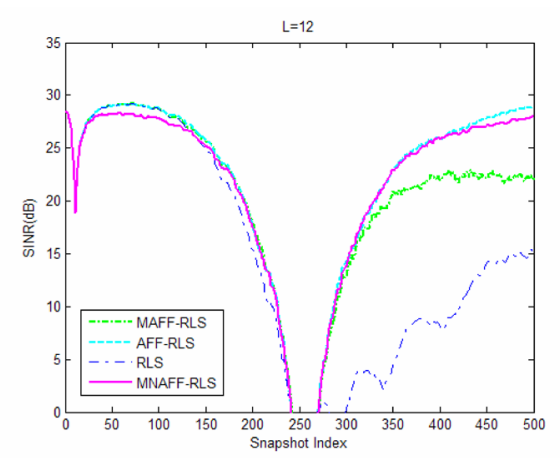

(c) The SINR result of each method

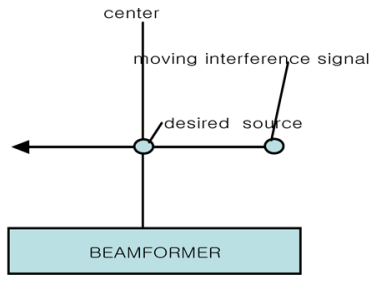

(b) The simulation scenario

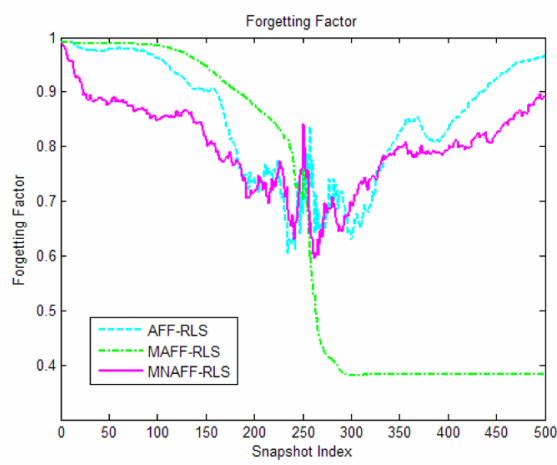

(d) The change of the forgetting factor

Fig. 1. The simulation settings and results. 